\title{
Influence of Thermodynamic Effect on Blade Load in a Cavitating Inducer
}

\author{
Kengo Kikuta, ${ }^{1}$ Noriyuki Shimiya, ${ }^{1}$ Tomoyuki Hashimoto, ${ }^{2}$ Mitsuru Shimagaki, ${ }^{2}$ \\ Hideaki Nanri, ${ }^{2}$ and Yoshiki Yoshida ${ }^{2}$ \\ ${ }^{1}$ IHI Corporation, 229, Tonogaya, Mizuho-machi, Nishitama-gun, Tokyo 190-1297, Japan \\ ${ }^{2}$ Japan Aerospace Exploration Agency (JAXA), Kakuda Space Center, Koganezawa 1, Kimigaya, Kakuda, Miyagi 981-1525, Japan
}

Correspondence should be addressed to Kengo Kikuta, kengo_kikuta@ihi.co.jp

Received 29 July 2010; Accepted 14 October 2010

Academic Editor: J.-C. Han

Copyright ( $) 2010$ Kengo Kikuta et al. This is an open access article distributed under the Creative Commons Attribution License, which permits unrestricted use, distribution, and reproduction in any medium, provided the original work is properly cited.

\begin{abstract}
Distribution of the blade load is one of the design parameters for a cavitating inducer. For experimental investigation of the thermodynamic effect on the blade load, we conducted experiments in both cold water and liquid nitrogen. The thermodynamic effect on cavitation notably appears in this cryogenic fluid although it can be disregarded in cold water. In these experiments, the pressure rise along the blade tip was measured. In water, the pressure increased almost linearly from the leading edge to the trailing edge at higher cavitation number. After that, with a decrease of cavitation number, pressure rise occurred only near the trailing edge. On the other hand, in liquid nitrogen, the pressure distribution was similar to that in water at a higher cavitation number, even if the cavitation number as a cavitation parameter decreased. Because the cavitation growth is suppressed by the thermodynamic effect, the distribution of the blade load does not change even at lower cavitation number. By contrast, the pressure distribution in liquid nitrogen has the same tendency as that in water if the cavity length at the blade tip is taken as a cavitation indication. From these results, it was found that the shift of the blade load to the trailing edge depended on the increase of cavity length, and that the distribution of blade load was indicated only by the cavity length independent of the thermodynamic effect.
\end{abstract}

\section{Introduction}

In rocket turbopump, due to the high speed and high flow rate, the employment of inducers is necessary to meet the suction head requirement for the main impeller. These inducers have high solidities to improve the cavitation performance, and their pressure rises basically depend on the angle of attack and the camber. In these inducers, the distribution of blade load gradually changes when cavitation occurs and develops. According to the development of cavitation, the high blade load area moves to the trailing edge of the inducer [1], which has been confirmed by experiments [2] and CFD [3]. Finally, the change of blade load affects the total inducer head. Thus, the blade load is one of the important characteristics of cavitating inducers [4].

On the other hand, the thermodynamic effect on cavitation is significant in cryogenic fluid. Because of the heat transfer for evaporation, saturated vapor pressure in the cavity decreases, and the cavity growth becomes smaller than that in the fluid in which the thermodynamic effect is negligible, that is, cold water. Therefore, the cavitation performance of the inducer is improved in cryogenic fluid. In our previous study $[5,6]$, the relation between the degree of the thermodynamic effect and the cavity length was examined by using liquid nitrogen as a working fluid.

The cavitation changes the distribution of blade load and also causes the thermodynamic effect in cryogenic fluid. However, the relationship between the thermodynamic effect and the change of the blade load in cryogenic flow is poorly understood. In the present work, to clarify this relationship, we conducted experiments in both cold water and liquid nitrogen with a focus on the development of cavity length.

\section{Experimental Facilities and Procedure}

2.1. Experimental Facilities. In our present work, we carried out two types of experiments: experiments in cold water, in which the thermodynamic effect can be disregarded, and 


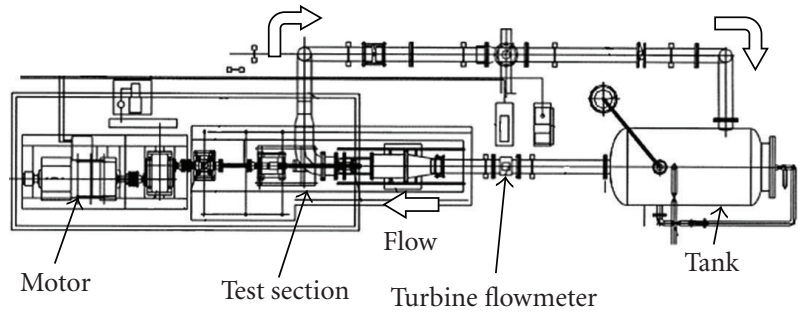

Figure 1: Inducer test facility of IHI.

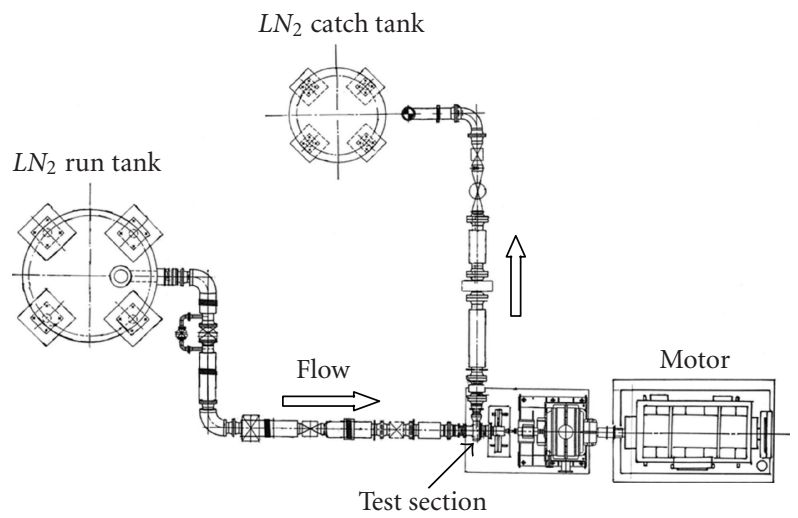

FIgURE 2: Cryogenic inducer test facility of JAXA.

experiments in liquid nitrogen, in which the thermodynamic effect appears distinctly. The inducers employed in both experiments had the same dimensions and geometric configurations (number of blades $Z=3$, solidity $C / h=$ about 2.0).

Experiments in water were conducted at the Inducer Test Facility of IHI Corporation, in the closed-loop cavitation tunnel shown in Figure 1 [7]. The working fluid was cold water at $306 \mathrm{~K}$ after degassing. The rotational speed was $N=$ $6000 \mathrm{rpm}$, and a flow rate was $\mathrm{Q} / \mathrm{Q}_{d}=1.00$.

Experiments in liquid nitrogen were conducted at the Cryogenic Inducer Test Facility (CITF) at Kakuda Space Center (KSC) of the Japan Aerospace Exploration Agency (JAXA) shown in Figure 2 [8]. The temperature of the liquid nitrogen was $79 \mathrm{~K}$. The rotational speed was set at $10000 \mathrm{rpm}$ with a flow rate of $Q / Q_{d}=1.00$.

These two experiments were conducted on different rotational speeds (water $/ 6000 \mathrm{rpm}$, nitrogen $/ 10000 \mathrm{rpm}$ ). It can be considered that when the flow rates $Q / Q_{d}$ are the same in these two experiment, their distribution of blade load are similar because their Reynolds number $(\mathrm{Re}=\mathrm{UC} / \nu)$ is large enough $\left(\operatorname{Re}>10^{7}\right)$.

2.2. Experimental Procedure. To measure the distribution of blade load, several pressure sensors were installed on the casing wall both in cold water and in liquid nitrogen. Figure 3 shows the location of the pressure taps along the blade. In the experiment in water, six sensors were installed $(W 1-W 6)$, and in the experiment in liquid nitrogen, three sensors were installed (N1-N3). Unfortunately, because of the limit of the test apparatus, these sensors could not be installed to the same locations in each experiment. The pressure rise along the blade tip was measured by these sensors.

We also focused on the development of cavity length. The cavity length $L_{c}$ of the tip leakage vortex cavitation along the blade was taken to indicate the cavitating situation since tip cavitations are considered to play important roles in cavitation performances of inducers. The cavity length $L_{c}$ was defined as the length from the leading edge of the blade to the trailing edge of the cavity. In the experiments, the cavitating length $L_{c}$ was estimated by the same procedure as that employed in a previous study [5]. In both the experiment in cold water and that in liquid nitrogen, the cavitating region was estimated based on the pressure distribution, which was obtained from the pressure waveform. Figure 4 shows the waterfalls of typical waveforms measured at unsteady sensor. Waveforms of 50 rotations are averaged using the trigger signal of a rotation. When the cavitation number becomes smaller, a flat region (light blue) appears at the bottom of the waveform. The pressure of this region can be considered to be the vapor pressure of the cavitation. Thus, we judged this domain to be the region of cavitation [5].

To estimate the cavity region clearly, other unsteady pressure sensors were installed in liquid nitrogen experiment. Figure 5 shows the location of these unsteady pressure taps (Pos.1-8) in liquid nitrogen. They are located from the leading edge to the trailing edge along the blade.

In addition, the casing was made of transparent acrylic resin in the experiment in cold water, which allowed us direct visual observation of the cavitating situation during pressure measurements.

Figure 6 shows the cavitating situation in the inducer by direct visual observation in cold water and pressure distribution in cold water and liquid nitrogen. In the images obtained by direct observation, the cavitating regions appear at the tip of the inducer. In the pressure distributions, the estimated cavitating regions are indicated in blue. It can be seen that these blue areas extend from the leading edge to the trailing edge corresponding to the cavitation observed in the images [9].

\subsection{Experimental Condition for the Thermodynamic Effect.} Although the thermodynamic effect on cavitation can be disregarded in cold water, it clearly appears in cryogenic fluid, The thermodynamic function $\Sigma\left(\mathrm{m} / \mathrm{s}^{3 / 2}\right)$ (see (1)), which was originally introduced by Brennen [10], is used as a parameter to estimate the degree of the thermodynamic effect. The thermodynamic function $\Sigma$ consists of thermal properties and depends only on fluid temperature in the same fluid

$$
\Sigma=\frac{\left(\rho_{v} L\right)^{2}}{\rho_{\ell}^{2} c_{p_{\ell}} T_{\infty} \sqrt{\alpha_{\ell}}}=\frac{\rho_{v} L}{\rho_{\ell}^{2} c_{p_{\ell}} \sqrt{\alpha_{\ell}}} \frac{d p_{v}}{d T} .
$$

Figure 7 shows thermodynamic function $\Sigma$ for nitrogen and water with a nondimensional temperature $T / T_{c}$, where $T_{c}$ is the temperature of the critical point. In addition, points for experimental conditions (nitrogen/79 K (०), water/306 K $(\bullet))$ are also plotted in this figure. 


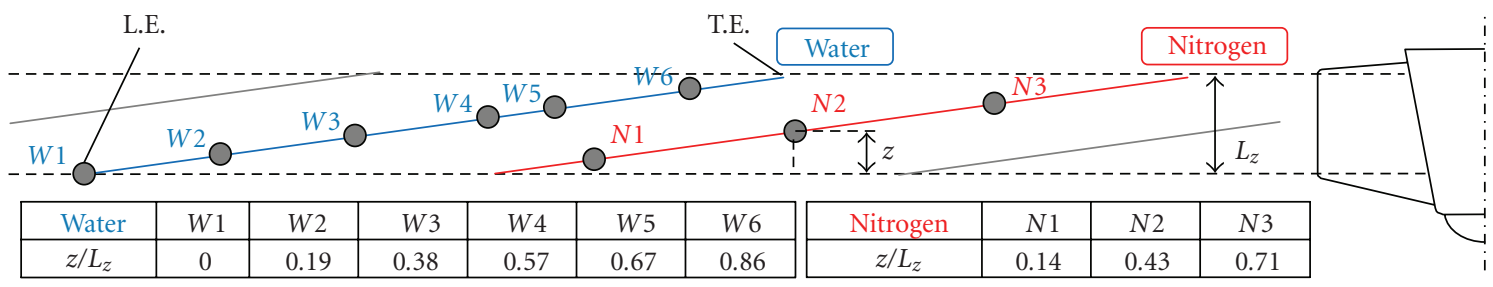

FIGURE 3: Development view of the inducer showing location of pressure sensors for measurements of pressure rise.

TABLE 1: Comparison of the thermodynamic parameter $\Sigma, \Sigma^{*}$.

\begin{tabular}{lccccc}
\hline & Temperature $T(\mathrm{~K})$ & Rotation $N(\mathrm{rpm})$ & $\operatorname{Re}(-)$ & $\Sigma\left(\mathrm{m} / \mathrm{s}^{3 / 2}\right)$ & $\Sigma^{*}=\Sigma\left(C / U^{3}\right)^{1 / 2}(\mathrm{nondim})$. \\
\hline Water & 306 & 6000 & $1.91 \times 10^{7}$ & $1.5 \times 10^{1}$ & $7.0 \times 10^{-1}$ \\
Nitrogen & 79 & 10000 & $1.27 \times 10^{8}$ & $4.0 \times 10^{4}$ & $3.1 \times 10^{1}$ \\
\hline
\end{tabular}

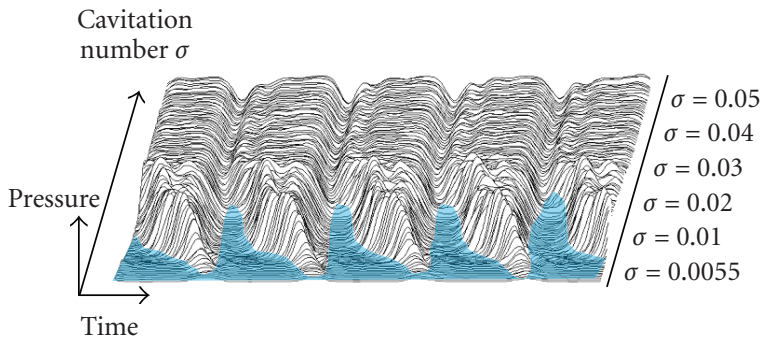

FIGURE 4: Waterfall of unsteady pressure waveform.

Watanabe et al. [11] introduced the nondimensional thermodynamic parameter $\Sigma^{*}$ (see (2)) on the twodimensional cascade model which analyzes the thermodynamic effect in a cavitating inducer

$$
\Sigma^{*}=\Sigma \sqrt{\frac{C}{U^{3}}} .
$$

Comparisons of the nondimensional thermodynamic parameter $\Sigma^{*}$ in the experiment in cold water with that in liquid nitrogen are shown in Table 1 . The value of $\Sigma^{*}(\Sigma)$ in the liquid nitrogen experiment $\left(\Sigma^{*}=31, \Sigma=4.0 \times 10^{4}\right)$ was much larger than that in cold water $\left(\Sigma^{*}=0.70, \Sigma=1.5 \times\right.$ $\left.10^{1}\right)$. Thus, the thermodynamic effect is expected to be much larger in the case of liquid nitrogen than water. The influence of velocity (rotational speed) on the thermodynamic effect was considered in this $\Sigma^{*}$, and we also investigated the influence in previous study [6].

\section{Experimental Result}

Figure 8 shows the pressure rise $\left(\psi_{i} / \psi_{o}\right)$ at the inducer outlet and at each pressure sensor shown in Figure 3. The result in cold water is shown at the top (a) in Figure 3, and that in liquid nitrogen is shown at the bottom (b) in Figure 3. In addition, the cavity length $C_{\mathrm{cl}}\left(=L_{c} / h\right.$, cavity length/blade spacing) is shown in the vertical axis on the right side. The horizontal axis is the cavitation number $\left(\sigma_{x} /\left(\sigma_{x}\right)_{0}\right)$, where $\left(\sigma_{x}\right)_{0}$ is the head break cavitation number of the experiment in cold water.
The inducer head (exit) in liquid nitrogen was maintained until the cavitation numbers became smaller than that in water. Thus, the cavitation performances were greatly improved in liquid nitrogen.

In both experiments, the cavity length $C_{\mathrm{cl}}$ increased almost linearly with the decrease of the cavitation number before the head break. After that, in both of the experiments, the cavity length $C_{\mathrm{cl}}$ grew rapidly ("jump") in the same $C_{\mathrm{cl}}$ region from 1.35 to 1.70 , and the head break occurred concurrently. Since this $C_{\mathrm{cl}}$ region $(1.35 \sim 1.70)$ was unrelated to the working fluid, this "jump" is considered to be a characteristic of the inducer which was employed in these experiments. On the other hand, the cavitation number when the "jump" of cavity length occurred was according to the working fluid. Thus, the thermodynamic effect appeared due to the suppression of cavity length, resulting in improvement of the cavitation performance, in liquid nitrogen.

From the inlet side, the pressure rise decreased with the decrease of the cavitation number. For example, in the experiment in liquid nitrogen, when $N 2$ (which was located on the inlet side) began to decrease $\left(\sigma_{x} /\left(\sigma_{x}\right)_{0}=\right.$ about 1.5$), N 3$ (which was located on the outlet side) became larger. That means that when the blade load around N2 decreased, that around N3 increased; that is, the high blade load area moved from the leading edge to the trailing edge of the inducer.

Furthermore, when the cavity length $C_{\mathrm{cl}}$ grew rapidly, pressure rise decreased concurrently in all of the sensors (W3 - $-W 6, N 2-N 3$ ). After rapid growth of the cavity length $C_{\mathrm{cl}}$, the cavity length grew slowly in liquid nitrogen in contrast to cold water. At that time, the decrease of pressure rise of $N 3$ was eased.

\section{Transition of Blade Load}

Because the blade load causes pressure to rise, the mean pressure gradient between two adjacent pressure sensors can be considered as average blade load in the area. To obtain the gradient of pressure rise, the pressure rise $\Delta\left(\psi_{i} / \psi_{o}\right)$ was divided by the axial location $\Delta\left(z / L_{z}\right)$ of each sensor. Figure 9 shows the mean pressure gradient $\Delta\left(\psi_{i} / \psi_{o}\right) / \Delta\left(z / L_{z}\right)$ of the experiment in liquid nitrogen. The gradient of pressure rise 


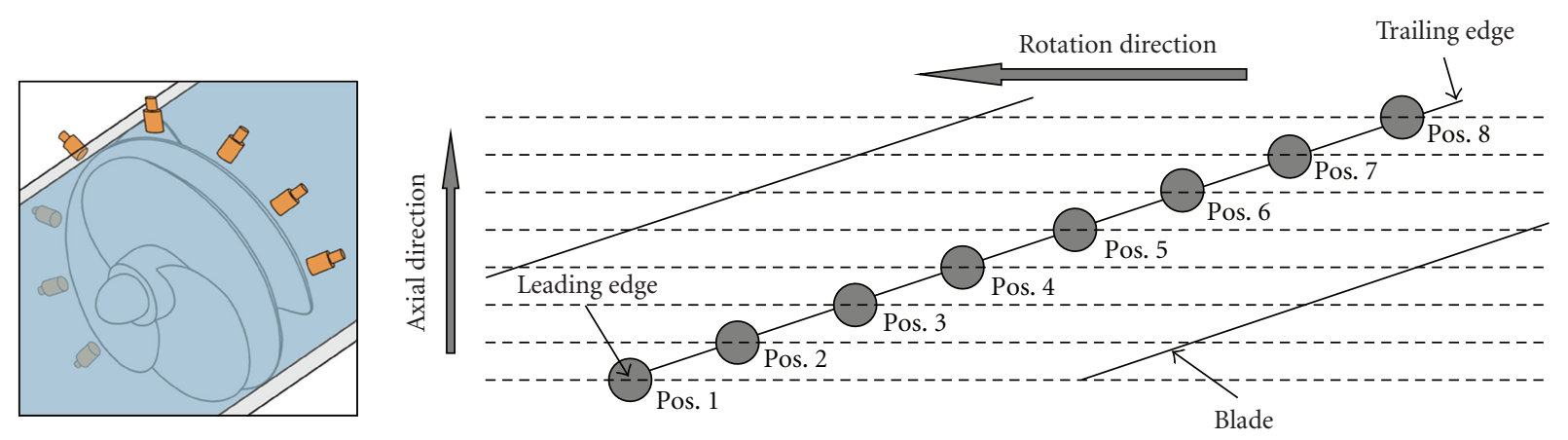

FIGURE 5: Location of pressure taps along the blade for measurements of unsteady pressure.

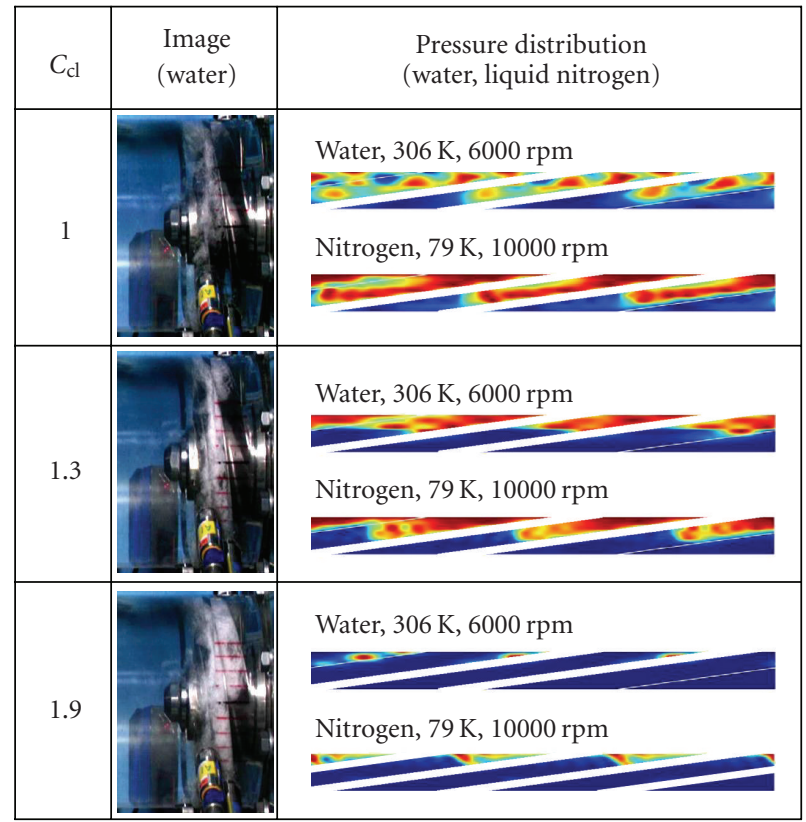

FIGURE 6: Visualization of cavity with direct images and indirect pressure distributions.

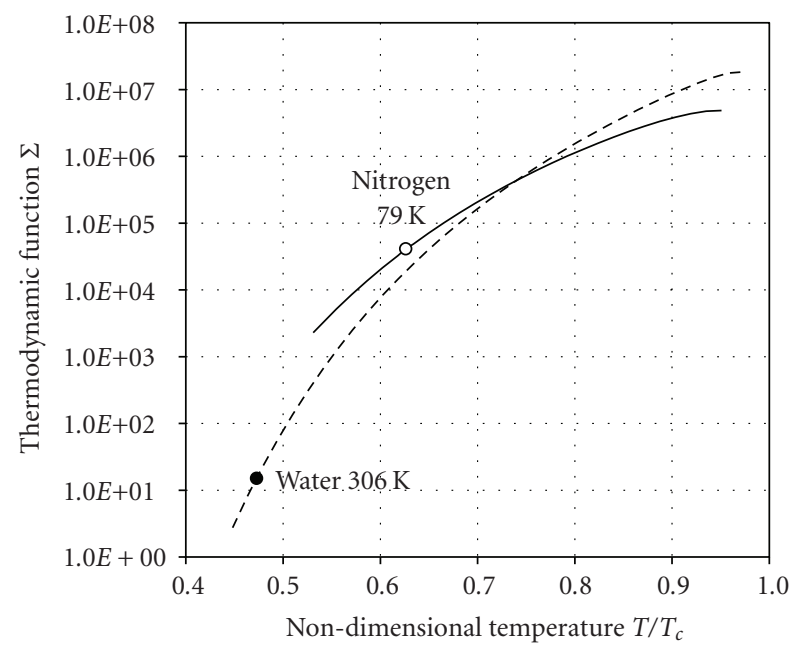

FIgURE 7: Comparison of the thermodynamic function $\Sigma(T)$ of nitrogen and water. is shown in the vertical axis. As the horizontal axis, the cavity length $C_{\mathrm{cl}}$ is used because the pressure rise is strongly related to the cavity length.

The blade load changes with the cavity length $C_{\mathrm{cl}}$. Before the cavity extends to the throat $\left(C_{\mathrm{cl}}<1.0\right)$, the gradient of the inlet part $(N 1 \rightarrow N 2)$ is largest. When the cavity length is $C_{\mathrm{cl}}=1.0$, the blade load occurring in the intermediate part $(N 2 \rightarrow N 3)$ increases instead of a decrease of the blade load of the inlet part $(N 1 \rightarrow N 2)$. After that, the cavity length $C_{\mathrm{cl}}$ ranges from 1.35 to 1.70 , though the plots are poor due to the rapid growth of cavity; the cavity seems to expand in the entire inlet area, and the blade load there $(N 1 \rightarrow N 2)$ approaches almost 0 . On the other hand, the blade load in the intermediate part $(N 2 \rightarrow N 3)$, and that in the outlet part (N3 $\rightarrow$ exit) increase. Thus, it is confirmed that the high blade load area moves to the trailing edge of the inducer according to the development of cavity length.

In addition, the gradient of pressure rise in the experiment in water $(W 3 \rightarrow W 5)$ is also shown in Figure 9. There is only one region shown due to the mismatch of the sensor location. This area $(W 3 \rightarrow W 5)$ is close to the same area of the intermediate part $(\mathrm{N} 2 \rightarrow \mathrm{N} 3)$ of the experiment in liquid nitrogen. Comparison of these corresponding areas shows that the gradient of pressure rise is almost the same at any cavity length. From this result, it is found that the transition of blade load is well shown by the cavity length with or without the thermodynamic effect.

\section{Influence of Cavity Length on Distribution of Blade Load}

Figure 10 shows pressure distribution from the leading edge $\left(z / L_{z}=0.0\right)$ to the trailing edge $\left(z / L_{z}=1.0\right)$ along the inducer tip.

Figure 10(a) shows the pressure distribution along the blade tip corresponding to the cavitation number $\sigma_{x} /\left(\sigma_{x}\right)_{0}$ as a cavitation parameter. In water, and at a higher cavitation number $\sigma_{x} /\left(\sigma_{x}\right)_{0}=3.0$ (indicated by $\mathbf{\square}$ ), the pressure increases almost linearly along the blade tip. After that, according to the cavitation number, it decreases (indicated by $\boldsymbol{\Lambda}$, and $\bullet$ ), and no pressure rise occurs in the inlet part, and a rise in pressure occurs drastically in a narrow area only near the trailing edge. On the other hand, in liquid nitrogen (indicated by $\square, \diamond, \Delta$, and $\circ$ ), the pressure distribution 

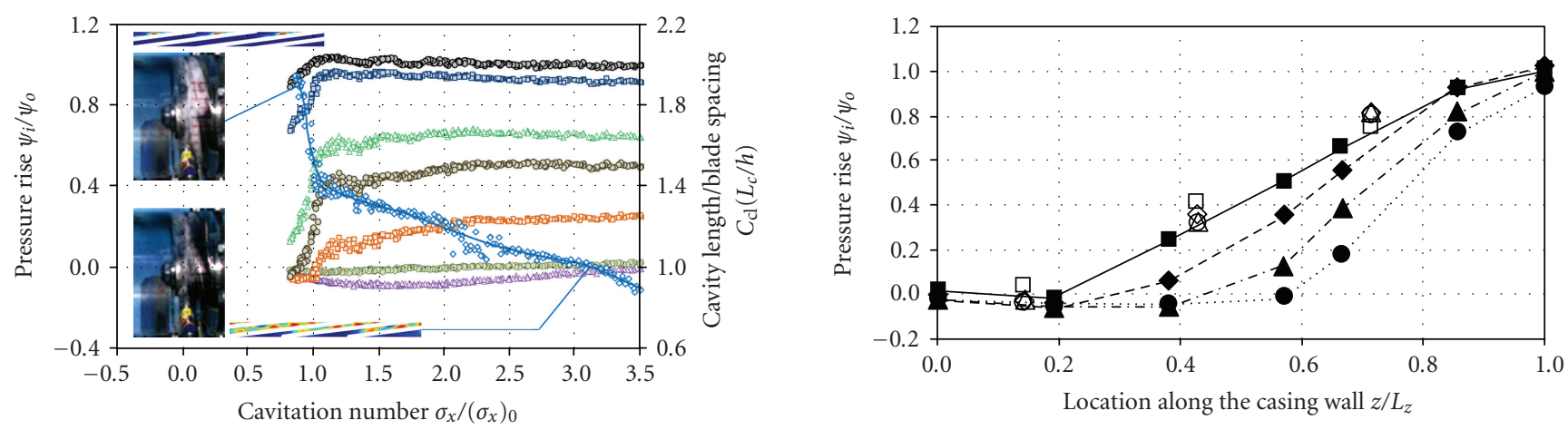

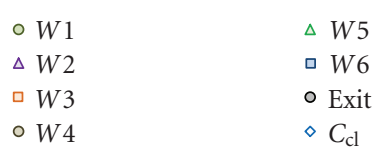

(a) Water
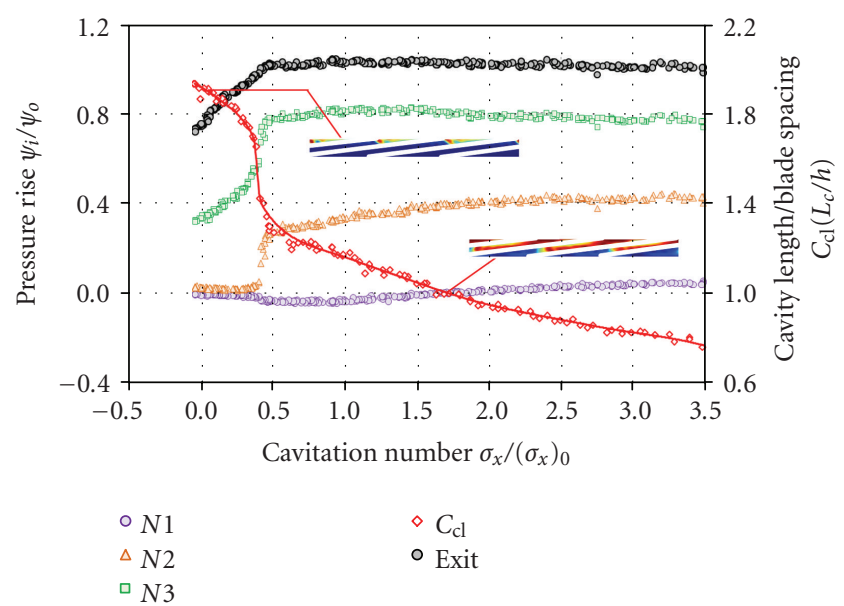

(b) Nitrogen

Figure 8: Pressure rise and cavity length in cold water (a) and in liquid nitrogen (b).

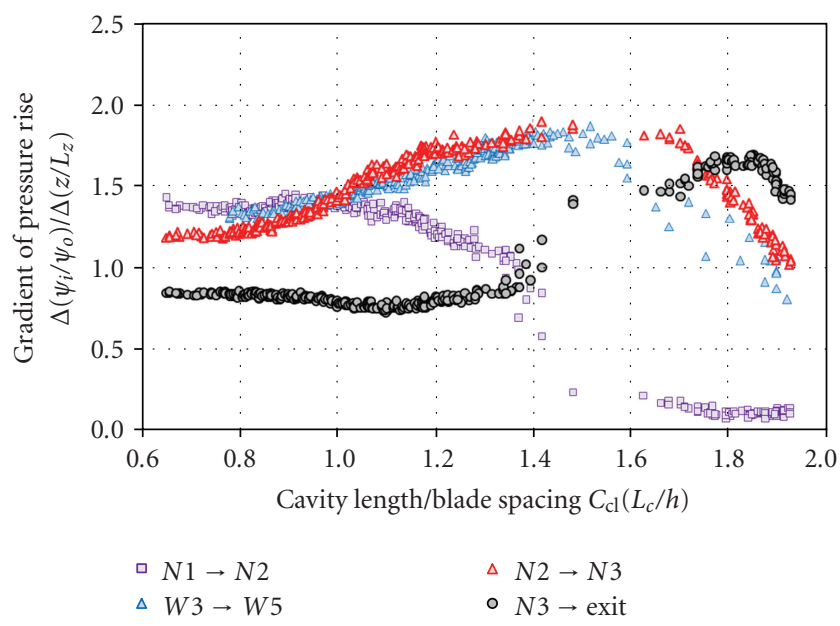

FIgURE 9: Comparison of the gradient of pressure rise between liquid nitrogen and water.

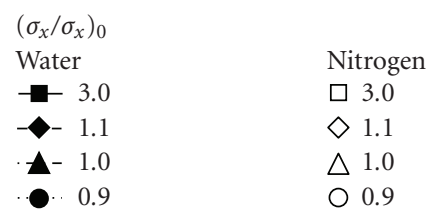

(a) Organized by cavitation number ratio $\sigma_{x} /\left(\sigma_{x}\right)_{0}$
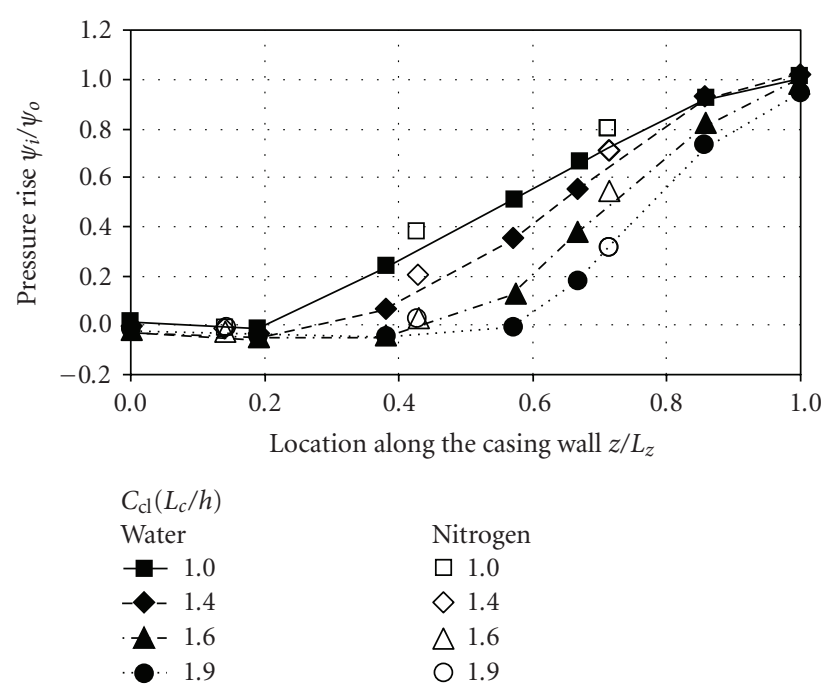

(b) Organized by cavity length $C_{\mathrm{cl}}\left(L_{c} / h\right)$

FIGURE 10: Pressure rise along the tip organized by cavitation number and cavity length.

is similar to that in water at a higher cavitation number $\sigma_{x} /\left(\sigma_{x}\right)_{0}=3.0$, even if the cavitation number decreases to $\sigma_{x} /\left(\sigma_{x}\right)_{0}=0.9$, where the blade load changes in water. Thus, the distribution of the blade load does not change even at lower cavitation numbers because the cavitation growth is suppressed by the thermodynamic effect.

Figure 10(b) shows the pressure distribution corresponding to the cavity length $C_{\mathrm{cl}}$. Comparison of the two experiments shows that the pressure distribution in liquid nitrogen has the same tendency as that in water. Thus, the distribution of blade load with the thermodynamic effect was quite similar to the one without the thermodynamic effect when the cavity length of tip cavitation $C_{\mathrm{cl}}$ is taken as a cavitation parameter.

As additional data to indicate the feature described above, a relationship between the pressure rise $\psi_{i} / \psi_{o}$, and the cavity length $C_{\mathrm{cl}}$ is shown in Figure 11. Both the pressure rises 


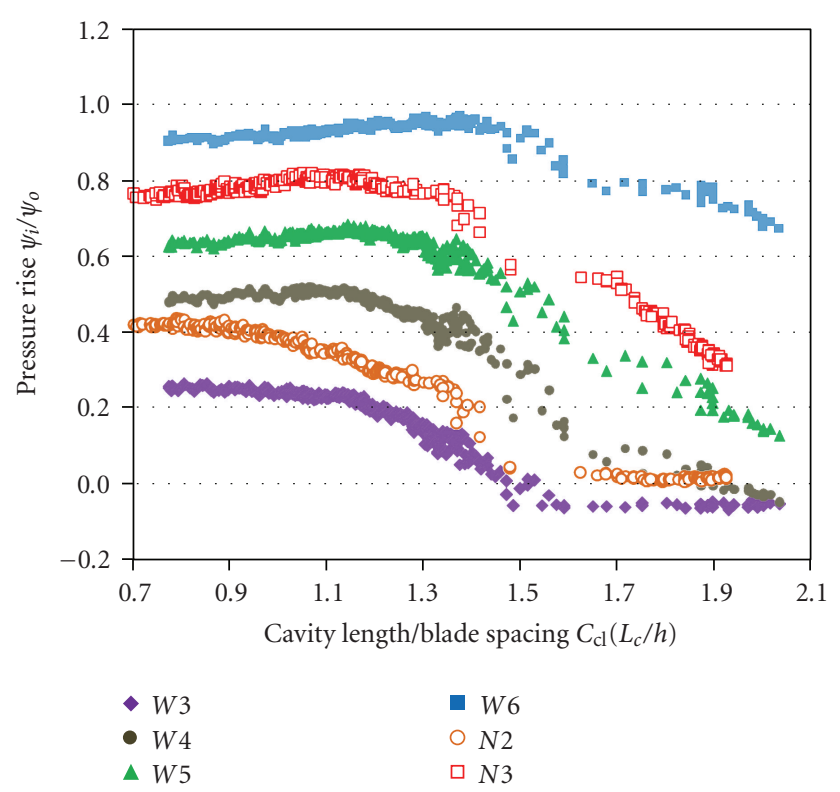

Figure 11: Comparison of the change of pressure rise between in liquid nitrogen and water.

$\psi_{i} / \psi_{o}$ in water (indicated by $\boldsymbol{\nabla}, \bullet, \boldsymbol{\Delta}$, and $\boldsymbol{\square}$ ) and those in liquid nitrogen (indicated by $\circ, \square$ ) are plotted in this figure. Although these pressure rises do not overlap each other because of the difference of the sensors locations (refer Figure 3), all of pressure raises $\psi_{i} / \psi_{o}$ decrease with increase of the cavity length $C_{\mathrm{cl}}$. Especially, even if the test fluid is different, the pressure rises in liquid nitrogen $N 2 / N 3$ are located between that in water $W 3$ and $W 4 /$ that in $W 5$ and $W 6$, in whole cavity length area. The order of these pressure levels corresponds to the order of sensor location. Thus, the distribution of pressure rise, which is caused by the blade load, has a similar tendency with or without the thermodynamic effect. Furthermore, that distribution changes depend only on the cavity length both in nitrogen and water.

From these results, it was found that the thermodynamic effect decreases the cavitation number in which the blade load is shifted to the trailing edge due to the suppression of the grows of cavity length. In addition, the distribution on blade load can be indicated only by the cavity length independent of the thermodynamic effect.

Stepanoff [12] investigated the thermodynamic effect on cavitation with regard to the reduction of pump performance (i.e., the measurable cavitation effect of $\Delta \psi / \psi_{0}=3 \%$ ) as an indication of cavitation, based on an assumption that "the same value of $V_{V} / V_{L}$ would mean the same extent of cavitation condition and the same damage to the performance" [13]. This was the pioneer work for the thermodynamic effect in pumps. By contrast, in the present work, it was found that "the same extent (length) of cavitation results in the same blade load distribution and results in the same damage to the performance in any working fluid." This is "converse" of Stepanoff's assumption, and it was confirmed in this work.

\section{Conclusion}

From experiments in water and liquid nitrogen for an investigation of thermodynamic effect on blade load, the following points were clarified.

(1) The thermodynamic effect decreases the cavitation number, in which the blade load is shifted to the trailing edge, due to the suppression of the growth of the cavity length.

(2) The distribution of blade load with the thermodynamic effect was almost similar to that without the thermodynamic effect in which the cavity length of tip cavitation is taken as a cavitation parameter. The distribution of blade load can be indicated by the cavity length as a cavitation parameter with/without the thermodynamic effect.

(3) The same extent (length) of cavitation results in the same blade load distribution and results in the same damage to the performance in any working fluid.

\section{Nomenclature}

\begin{tabular}{|c|c|}
\hline$C:$ & Chord of blade \\
\hline$C_{\mathrm{cl}}:$ & Cavity length/blade spacing $=L_{c} / h$ \\
\hline$c_{p l}:$ & Specific heat of liquid \\
\hline$h:$ & Spacing \\
\hline$L:$ & Latent heat \\
\hline$L_{z}:$ & Axial length of inducer blade in Figure 3 \\
\hline$L_{c}:$ & Cavity length \\
\hline$N:$ & Inducer rotational speed \\
\hline$p_{\nu}:$ & Vapor pressure \\
\hline Q: & Flow rate \\
\hline$Q_{d}:$ & Design flow rate \\
\hline Re: & Reynolds number $=\mathrm{UC} / \nu$ \\
\hline$T:$ & Temperature \\
\hline$T_{c}$ : & Cavity temperature \\
\hline$T_{\infty}:$ & Ambient temperature \\
\hline$U:$ & Tip velocity of inducer \\
\hline$V_{L}:$ & Volume of vapor \\
\hline$V_{V}:$ & Volume of liquid \\
\hline$Z:$ & Blade number \\
\hline$z$ : & Axial length of pressure sensor in Figure 3 \\
\hline$\alpha_{l}:$ & Thermal diffusivity of liquid \\
\hline$\rho_{l}:$ & Liquid density \\
\hline$\rho_{v}:$ & Vapor density \\
\hline$v:$ & Dynamic coefficient of viscosity \\
\hline$\Sigma:$ & Thermodynamic function defined by (1) \\
\hline$\Sigma^{*}:$ & $\begin{array}{l}\text { Nondimensional thermodynamic parameter } \\
\text { defined by (2) }\end{array}$ \\
\hline & Cavitation number \\
\hline$\left(\sigma_{x}\right)_{0}:$ & $\begin{array}{l}\text { Critical cavitation number in the absence of } \\
\text { any thermal effect (cold water) }\end{array}$ \\
\hline$\psi$ & Head coefficient \\
\hline 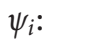 & Pressure rise coefficient at each sensors \\
\hline & Normal head coefficient. \\
\hline
\end{tabular}




\section{Acknowledgments}

The authors would like to thank IHI Corporation, which allowed use of the experimental data, and Mr. Satoshi Kawasaki of Japan Aerospace Exploration Agency (JAXA), who gave valuable advice.

\section{References}

[1] H. R. Badowski, "An explanation for instability in cavitating inducers," in Proceedings of the ASME Cavitation Forum, pp. 38-40, 1969.

[2] Y. Takamatsu, K. Ishizaka, T. Kishikawa, and Y. Kusaka, "An investigation of Tandem-Bladed herical inducer for cavitationresistant centrifugal pump," in Proceedings of the 10th IAHR Symposium, pp. 291-302, Tokyo, Japan, 1980.

[3] A. Hosangadi, V. Ahuja, and R. J. Ungewitter, "Simulations of cavitating flows in turbopumps," Journal of Propulsion and Power, vol. 20, no. 4, pp. 604-611, 2004.

[4] NASA, "Liquid Rocket Engine Turbopump Inducers," Space Vehicle Design Criteria SP-8052, 1971.

[5] Y. Yoshida, K. Kikuta, S. Hasegawa, M. Shimagaki, and T. Tokumasu, "Thermodynamic effect on a cavitating inducer in liquid nitrogen," Journal of Fluids Engineering, Transactions of the ASME, vol. 129, no. 3, pp. 273-278, 2007.

[6] K. Kikuta, Y. Yoshida, M. Watanabe, T. Hashimoto, K. Nagaura, and K. Ohira, "Thermodynamic effect on cavitation performances and cavitation instabilities in an inducer," Journal of Fluids Engineering, Transactions of the ASME, vol. 130, no. 11, pp. 1113021-1113028, 2008.

[7] K. Nakano and S. Kawasaki, "Inducer Test Facility," IIC REVIEW, No.39, pp. 73-76, 2008.

[8] Y. Yoshida, M. Watanabe, S. Hasegawa et al., "JAXA cryogenic inducer test facility," Turbomachinery, vol. 36-38, pp. 468-475, 2005 (Japanese).

[9] K. Kikuta, Y. Yoshida, T. Hashimoto, H. Nanri, T. Mizuno, and N. Shimiya, "Influence of rotational speed on thermodynamic effect in a cavitating inducer," in Proceedings of the ASME Fluids Engineering Division Summer Conference (FEDSM '09), pp. 77-83, Vail, Colo, USA, August 2009.

[10] C. Brennen, "The dynamic behavior and compliance of a stream of cavitating bubbles," Journal of Fluids Engineering, Transactions of the ASME, vol. 95, no. 4, pp. 533-541, 1973.

[11] S. Watanabe, T. Hidaka, H. Horiguchi, A. Furukawa, and Y. Tsujimoto, "Steady analysis of the thermodynamic effect of partial cavitation using the singularity method," Journal of Fluids Engineering, Transactions of the ASME, vol. 129, no. 2, pp. 121-127, 2007.

[12] A. J. Stepanoff, "Cavitation properties of liquids," ASME Journal of Engineering for Power, pp. 195-200, 1964.

[13] H. A. Stahl and A. J. Stepanoff, "Thermodynamic aspects of cavitation in centrifugal pumps," ASME Journal of Basic Engineering, vol. 78, pp. 1691-1693, 1956. 

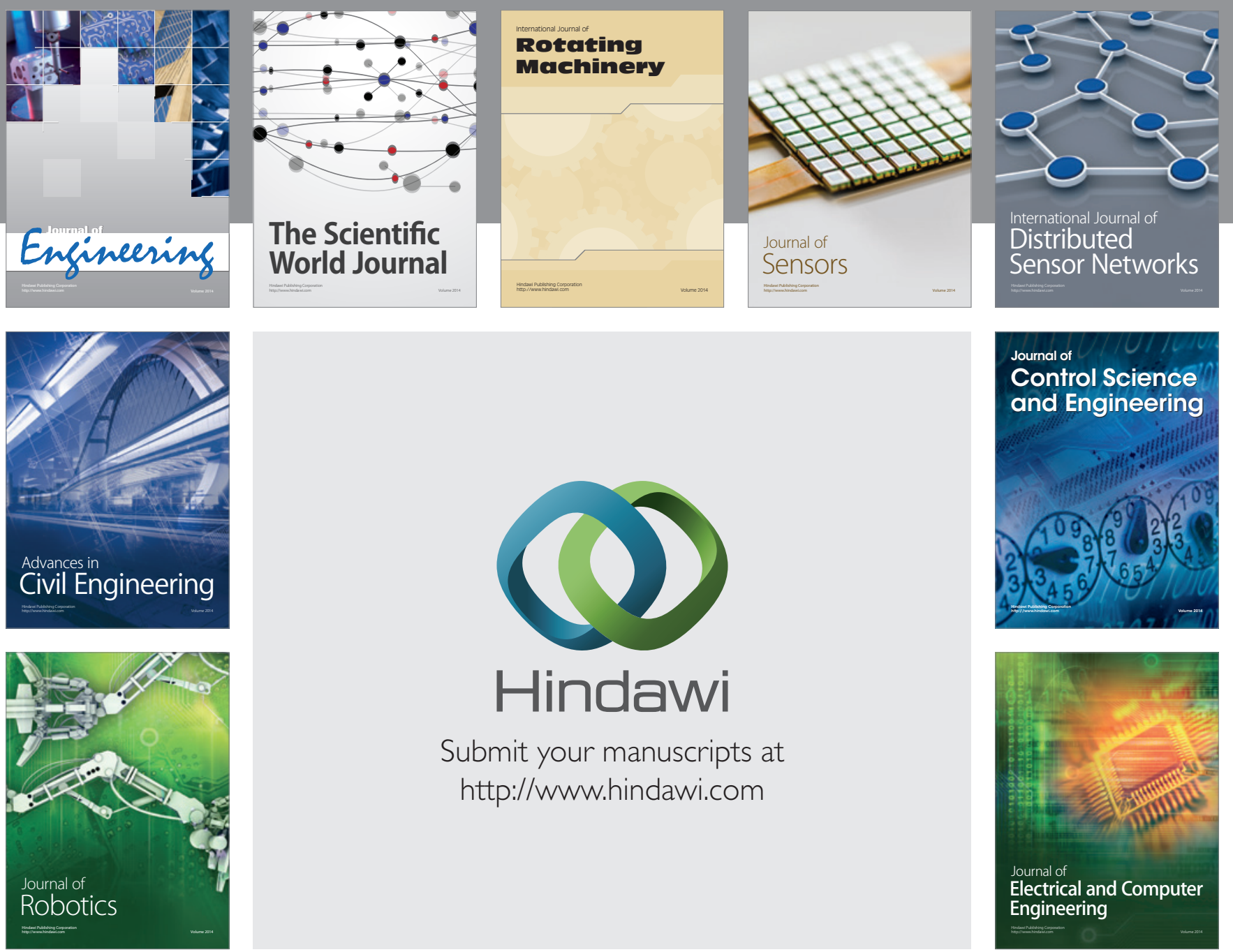

Submit your manuscripts at

http://www.hindawi.com
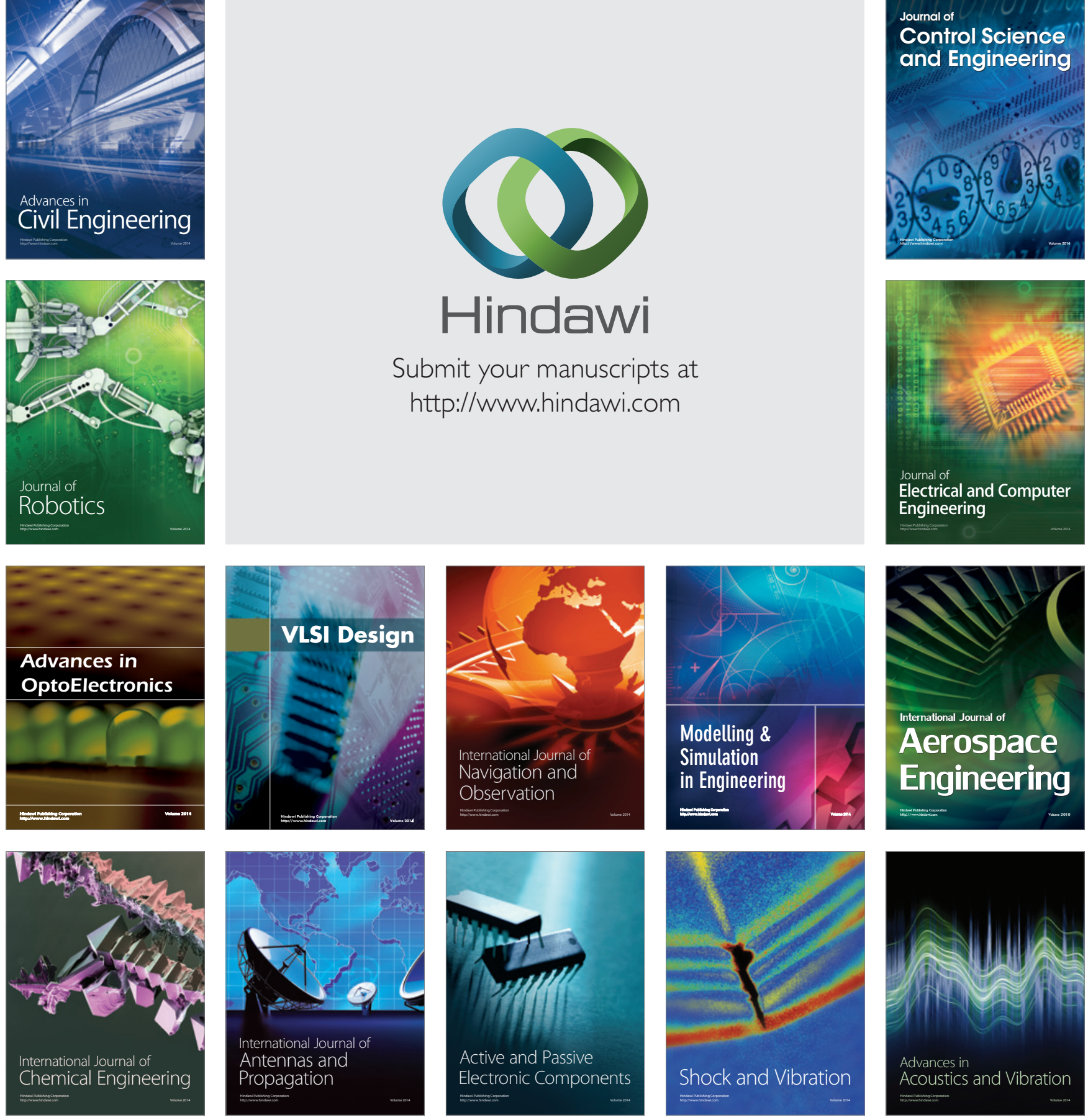